\title{
POWER REGULAR OPERATORS
}

\author{
AHARON ATZMON
}

\begin{abstract}
We show that for a wide class of operators $T$ on a Banach space, including the class of decomposable operators, the sequence $\left\{\left\|T^{n} x\right\|^{1 / n}\right\}_{n=1}^{\infty}$ converges for every $x$ in the space to the spectral radius of the restriction of $T$ to the subspace $\bigvee_{n=0}^{\infty}\left\{T^{n} x\right\}$.
\end{abstract}

\section{INTRODUCTION}

Throughout this paper, $X$ will denote a complex Banach space and $\mathscr{L}(X)$ the Banach algebra of bounded linear operators on $X$. For an operator $T$ in $\mathscr{L}(X)$, we denote as usual by $\sigma(T)$ its spectrum and by $r(T)$ its spectral radius. By Gelfand's formula for the spectral radius

$$
r(T)=\lim _{n \rightarrow \infty}\left\|T^{n}\right\|^{1 / n}
$$

for all $T$ in $\mathscr{L}(X)$.

It is well known that if $\left\{w_{n}\right\}_{n=1}^{\infty}$ is a sequence of nonnegative numbers which is submultiplicative (that is, $w_{m+n} \leq w_{m} w_{n}$ for all $m$ and $n$ ), then $\lim _{n \rightarrow \infty} w_{n}^{1 / n}$ exists. Hence, the existence of the limit in the right-hand side of Gelfand's formula can be deduced from the fact that for every $T$ in $\mathscr{L}(X)$, the sequence $\left\{\left\|T^{n}\right\|\right\}_{n=1}^{\infty}$ is submultiplicative. On the other hand, for $T$ in $\mathscr{L}(X)$ and $x$ in $X$, the sequence $\left\{\left\|T^{n} x\right\|\right\}_{n=1}^{\infty}$ is in general not submultiplicative. Nevertheless, we shall show in this paper that for a wide class of operators $T$ in $\mathscr{L}(X)$, the sequence $\left\{\left\|T^{n} x\right\|^{1 / n}\right\}_{n=1}^{\infty}$ is convergent for all $x$ in $X$. We shall call such operators power-regular.

We shall prove, in particular, that all decomposable operators (see definition in §3) are power-regular. By [4] this class includes all spectral operators in Dunford's sense (hence all normal operators) and all operators with totally disconnected (hence countable) spectrum. We shall also prove that every operator $T$ in $\mathscr{L}(X)$ for which the set $\{|\lambda| ; \lambda \in \sigma(T)\}$ has empty interior in $[0, \infty)$ is power-regular. This class clearly contains all operators with spectrum included in a countable union of circles with centers at the origin. Moreover, we shall show that for every operator $T$ in $\mathscr{L}(X)$ which belongs to one of these classes, the sequence $\left\{\left\|T^{n} x\right\|^{1 / n}\right\}_{n=1}^{\infty}$ converges for all $x$ in $X$ to the spectral radius of the restriction of $T$ to the subspace $\bigvee_{n=0}^{\infty}\left\{T^{n} x\right\}$.

Received by the editors February 28, 1994; originally communicated to the Proceedings of the $A M S$ by Palle E. T. Jorgensen.

1991 Mathematics Subject Classification. Primary 47A11; Secondary 47A35. 
Power-regularity of compact operators and selfadjoint operators can also be deduced from the results in [11, §9]. In [5] power-regularity is also established for a more general class of operators with countable spectrum and for normal operators.

We recall that the local spectral radius of an operator $T$ in $\mathscr{L}(X)$ at a vector $x$ in $X$ is defined by (cf. [5] and [13])

$$
r(x, T)=\limsup _{n \rightarrow \infty}\left\|T^{n} x\right\|^{1 / n} \text {. }
$$

As shown in [5], for every $T$ in $\mathscr{L}(X)$, the equality $r(x, T)=r(T)$ holds for quasi-all $x$ in $X$ (that is, for all $x$ in the complement of a set of first category). Thus if $T$ is power-regular,

$$
r(x, T)=\lim _{n \rightarrow \infty}\left\|T^{n} x\right\|^{1 / n}
$$

for all $x$ in $X$, and the limit is equal to $r(T)$ for quasi-all $x$ in $X$.

We mention also that by [13], for every $T$ in $\mathscr{L}(X),\left\{\left\|T^{n} x\right\|^{1 / n}\right\}_{n=1}^{\infty}$ converges to $r(T)$ for all $x$ in a dense subset of $X$. For Hilbert spaces this follows also from [3, Theorem 2.A.1]. On the other hand, it is proved in [5] that if for $T$ in $\mathscr{L}(X)$ and $x$ in $X$ the sequence $\left\{\left\|T^{n} x\right\|^{1 / n}\right\}_{n=1}^{\infty}$ does not converge, then its set of limit points is a closed interval.

It is easy to construct weighted shifts which are not power-regular (cf. [5] and [9]). We shall also see in $\S 6$ that the backward shift on $l^{2}$ is not power-regular.

In $\S 2$ we establish a general criterion for power-regularity from which most of our subsequent results are derived. In $\S 3$ we prove power-regularity of decomposable operators and deduce several corollaries. In $\S 4$ we introduce the class of radially decomposable operators and prove power-regularity of operators belonging to the subclass of radially super-decomposable operators. We show that this subclass contains all operators $T$ in $\mathscr{L}(X)$ for which the set $\{|\lambda| ; \lambda \in \sigma(T)\}$ has empty interior in $[0, \infty)$. In $\S 5$ we give two direct and elementary proofs of power-regularity of operators on a finite-dimensional space. Finally, in $\S 6$, we present some additional facts and examples and raise several problems.

\section{A GENERAL CRITERION}

In this section we prove a general criterion for power-regularity which is applied in the sequel to establish power-regularity of operators in some concrete classes, in particular in those mentioned in the previous section. We first need some notation.

Let $T$ be an operator in $\mathscr{L}(X)$. We denote as usual by $\operatorname{Lat}(T)$ the collection of all closed subspaces of $X$ which are invariant under $T$, and for a subspace $M$ in $\operatorname{Lat}(T)$ we denote by $T_{M}$ the restriction of $T$ to $M$. We shall denote by $q(T)$ the minimum of the set $\{|\lambda| ; \lambda \in \sigma(T)\}$ (for $X=\{0\}$, we define $q(T)=\infty)$. For every $x$ in $X$ we shall denote by $r_{x}(T)$ the spectral radius of the restriction of $T$ to the subspace $\bigvee_{n=0}^{\infty}\left\{T^{n} x\right\}$ (for $x=0$, we define $\left.r_{x}(T)=0\right)$. Note that by the spectral radius formula,

$$
r(x, T) \leq r_{x}(T) \leq r(T)
$$

for all $x$ in $X$.

We can now state our general criterion for power-regularity. 
Theorem 2.1. Let $T$ be an operator in $\mathscr{L}(X)$, and assume that for every $0<$ $t_{1}<t_{2}<\infty$ there exist a complex Banach space $Y$, an operator $S$ in $\mathscr{L}(Y)$, and a bounded linear operator $J$ from $X$ into $Y$, such that setting $M=\operatorname{ker} J$, the following three conditions hold:

(I) $J T=S J$;

(II) $r\left(T_{M}\right) \leq t_{2}$

(III) $q(S) \geq t_{1}$.

Then for all $x$ in $X$,

$$
\lim _{n \rightarrow \infty}\left\|T^{n} x\right\|^{1 / n}=r_{x}(T) .
$$

Remarks. (1) Condition (I) implies that $M \in \operatorname{Lat}(T)$.

(2) For $t_{2}>r(T)$ the above conditions hold for every $T$ in $\mathscr{L}(X)$, with $Y=X, S=t_{2} I$, and $J=0$.

The following fact is needed for the proof of the theorem.

Lemma 2.2. If $Y$ is a complex Banach space and $S$ is an operator in $\mathscr{L}(Y)$, then for every $y \neq 0$ in $Y$,

$$
q(S) \leq \liminf _{n \rightarrow \infty}\left\|T^{n} y\right\|^{1 / n} .
$$

Proof. The inequality is clear if $q(S)=0$. Assume that $q(S)>0$. Then $S$ is invertible and $r\left(S^{-1}\right)=(q(S))^{-1}$. Hence, noticing that for every $y$ in $Y$

$$
\|y\| \leq\left\|S^{-n}\right\|\left\|S^{n} y\right\|, \quad n=1,2, \ldots,
$$

by using the spectral radius formula for $S^{-1}$ we obtain that if $y \neq 0$,

$$
1=\lim _{n \rightarrow \infty}\|y\|^{1 / n} \leq(q(S))^{-1} \liminf _{n \rightarrow \infty}\left\|S^{n} y\right\|^{1 / n},
$$

and the lemma is proved.

Proof of Theorem 2.1. Let $x \in X$. If $r_{x}(T)=0$ the assertion is clear. Assume that $r_{x}(T)>0$, and consider numbers $t_{1}$ and $t_{2}$ such that $0<t_{1}<t_{2}<r_{x}(T)$. Let $S$ and $J$ be the operators which satisfy the conditions of the theorem for $t_{1}$ and $t_{2}$. We claim that $J x \neq 0$. In fact, assuming that $x \in M=\operatorname{ker} J$ and remembering that $M \in \operatorname{Lat}(T)$, we obtain that $\bigvee_{n=0}^{\infty}\left\{T^{n} x\right\} \subset M$, and therefore $r_{x}(T) \leq r\left(T_{M}\right)$, which by condition (II) contradicts the choice of $t_{2}$. So $J x \neq 0$ (and in particular $J \neq 0$ ). Thus by Lemma 2.2 and condition (III),

$$
t_{1} \leq q(S) \leq \liminf _{n \rightarrow \infty}\left\|S^{n} J x\right\|^{1 / n} .
$$

Therefore, observing that by condition (I),

$$
J T^{n} x=S^{n} J x, \quad n=1,2, \ldots,
$$

we obtain that

$$
t_{1} \leq \liminf _{n \rightarrow \infty}\left\|S^{n} J x\right\|^{1 / n} \leq \liminf _{n \rightarrow \infty}\left(\|J\|^{1 / n}\left\|T^{n} x\right\|^{1 / n}\right)=\liminf _{n \rightarrow \infty}\left\|T^{n} x\right\|^{1 / n} ;
$$

and since $t_{1}$ is an arbitrary number less than $r_{x}(T)$, we deduce that

$$
r_{x}(T) \leq \liminf _{n \rightarrow \infty}\left\|T^{n} x\right\|^{1 / n} .
$$

But as noticed at the beginning of this section,

$$
r(x, T)=\limsup _{n \rightarrow \infty}\left\|T^{n} x\right\|^{1 / n} \leq r_{x}(T),
$$


and we conclude that

$$
\lim _{n \rightarrow \infty}\left\|T^{n} x\right\|^{1 / n}=r_{x}(T) .
$$

This completes the proof of the theorem.

\section{DECOMPOSABLE OPERATORS}

In this section we shall establish power-regularity of decomposable operators. We recall that according to [1] an operator $T$ in $\mathscr{L}(X)$ is decomposable if for every cover of the complex plane by a pair of open sets $U$ and $V$, there exist subspaces $M$ and $K$ in $\operatorname{Lat}(T)$ such that $M+K=X, \sigma\left(T_{M}\right) \subset U$, and $\sigma\left(T_{K}\right) \subset V$.

The main ingredients in the proof of power-regularity of decomposable operators are Theorem 2.1 in the preceding section and Theorem 12.15 in [7].

In the proof we shall need an additional notation. For an operator $T$ in $\mathscr{L}(X)$ and a subspace $M$ in $\operatorname{Lat}(T)$, we shall denote by $T^{M}$ the canonical operator induced by $T$ on the quotient space $X / M$.

Theorem 3.1. If $T$ is a decomposable operator in $\mathscr{L}(X)$, then for all $x$ in $X$,

$$
\lim _{n \rightarrow \infty}\left\|T^{n} x\right\|^{1 / n}=r_{x}(T) \text {. }
$$

Proof. Let $0<t_{1}<t_{2}<\infty$, and consider the disc $G=\left\{\lambda \in \mathbb{C} ;|\lambda|<t_{2}\right\}$. Since $T$ is decomposable, it follows from [7, Theorem 12.15] that there exists a subspace $M$ in $\operatorname{Lat}(T)$, such that $\sigma\left(T_{M}\right) \subset \bar{G}$ and $\sigma\left(T^{M}\right) \cap G=\varnothing$. This is equivalent to the conditions $r\left(T_{M}\right) \leq t_{2} \leq q\left(T^{M}\right)$. From this it is readily verified that the conditions of Theorem 2.1 hold for $t_{1}$ and $t_{2}$, with $Y=$ $X / M, S=T^{M}$, and $J$ the canonical map of $X$ onto $X / M$, and the proof is complete.

It follows from Theorem 3.1 that all operators considered in [4] are powerregular. In particular, from [4, pp. 33, 67, and 185] we obtain the following.

Corollary 3.2. If $T$ is an operator in $\mathscr{L}(X)$, then each of the following conditions implies that the conclusion of Theorem 3.1 holds for $T$.

(1) $T$ is a spectral operator in Dunford's sense (hence, in particular, if $T$ is a normal operator in Hilbert space).

(2) $\sigma(T)$ is totally disconnected (hence, in particular, if $\sigma(T)$ is countable).

(3) $\sigma(T)$ is included in the real line, and the integral

$$
\int_{0}^{1} \ln \ln \sup _{|\operatorname{Im} \lambda|>y}\left\|(T-\lambda I)^{-1}\right\| d y
$$

is convergent. (This condition is satisfied, in particular, if $\left\|(T-\lambda I)^{-1}\right\| \leq$ $c \exp \left(b /|\operatorname{Im} \lambda|^{\sigma}\right)$, for $\operatorname{Im} \lambda \neq 0$, where $\alpha, b, c$ are positive constants.)

Another corollary is concerned with Banach algebras. We shall say that a Banach algebra $B$ is power-regular, if $\lim _{n \rightarrow \infty}\left\|x^{n} y\right\|^{1 / n}$ exists for all $x$ and $y$ in $B$.

The following is an immediate consequence of Theorem 3.1 and [4, Theorem 2.6, p. 201]. 
Corollary 3.3. Every commutative semi-simple regular Banach algebra is powerregular.

\section{RADIALLY DECOMPOSABLE OPERATORS}

According to [7, Theorem 12.5], if $T$ is a decomposable operator, then for every open set $G$ in the complex plane, there exists a subspace $M$ in $\operatorname{Lat}(T)$ such that $\sigma\left(T_{M}\right) \subset \bar{G}$ and $\sigma\left(T^{M}\right) \cap G=\varnothing$. In the proof of Theorem 3.1 we used only the fact that this holds when $G$ is an open disc with center at the origin. This suggests that power-regularity might be true for operators $T$ in $\mathscr{L}(X)$ which satisfy a condition that is considerably weaker than decomposability, namely, that for every $0<t_{1}<t_{2}<\infty$, there exists subspaces $M$ and $K$ in $\operatorname{Lat}(T)$, such that $M+K=X, q\left(T_{M}\right) \geq t_{1}$, and $r\left(T_{K}\right) \leq t_{2}$. We shall call operators which satisfy this condition radially decomposable.

We conjecture that all radially decomposable operators are power-regular. This would follow from the proof of Theorem 3.1, if one could show that the conclusion of Theorem 12.15 in [7] holds for these operators for open discs with centers at the origin.

We prove in this section power-regularity of operators which belong to a somewhat more restricted class. Before describing it, we mention that, according to [12], an operator $T$ in $\mathscr{L}(X)$ is called super-decomposable if for every cover of the complex plane by a pair of open sets $U$ and $V$, there exists an operator $A$ in $\mathscr{L}(X)$ which commutes with $T$ such that $\sigma\left(T_{\overline{A X}}\right) \subset U$ and $\sigma(T \overline{(I-A) X}) \subset V$.

Motivated by this terminology, we shall say that an operator $T$ in $\mathscr{L}(X)$ is radially super-decomposable if for every $0<t_{1}<t_{2}<\infty$, there exists an operator $A$ in $\mathscr{L}(X)$ which commutes with $T$ such that $q\left(T_{\overline{A X}}\right) \geq t_{1}$ and $r\left(T_{\overline{(I-A) X}}\right) \leq t_{2}$.

It is clear that a radially super-decomposable operator is radially decomposable and that a super-decomposable operator is decomposable and radially super-decomposable.

Theorem 4.1. If $T$ is a radially super-decomposable operator in $\mathscr{L}(X)$, then for all $x$ in $X$,

$$
\lim _{n \rightarrow \infty}\left\|T^{n} x\right\|^{1 / n}=r_{x}(T)
$$

Proof. Let $0<t_{1}<t_{2}<\infty$, and consider an operator $A$ in $\mathscr{L}(X)$ which commutes with $T$ such that $q\left(T_{\overline{A X}}\right) \geq t_{1}$ and $r\left(T_{\overline{(I-A) X}}\right) \leq t_{2}$. Observing that $\operatorname{ker} A \subset(I-A) X$, we see that $r\left(T_{\operatorname{ker} A}\right) \leq r\left(T_{\overline{(I-A) X}}\right)$; and this implies that the conditions of Theorem 2.1 hold for $t_{1}$ and $t_{2}$, with $Y=\overline{A X}, S=T_{Y}$, and $J=A$. This completes the proof.

Remark. It follows from [12, Propositions 2.1 and 2.2] that all operators that satisfy the conditions of Corollary 3.2 are super-decomposable, and therefore the corollary also follows from Theorem 4.1. By [12, Corollary 2.4] the same is true for Corollary 3.3.

Theorem 4.2. If $T$ is an operator in $\mathscr{L}(X)$ such that the set $\{|\lambda| ; \lambda \in \sigma(T)\}$ has empty interior in $[0, \infty)$, then the conclusion of Theorem 4.1 holds for $T$. Proof. We shall show that $T$ is radially super-decomposable. Let $0<t_{1}<$ $t_{2}$, and consider the set $B=\{|\lambda| ; \lambda \in \sigma(T)\}$. Since $B$ has empty interior 
in $[0, \infty)$, there exist $t \notin B$ such that $t_{1}<t<t_{2}$, and therefore the set $\tau=\{\lambda \in \mathbb{C} ;|\lambda| \geq t\} \cap \sigma(T)$ is a spectral set for $T$ (that is, an open and closed subset of $\sigma(T))$. Let $A$ denote the corresponding spectral projection $E(\tau, T)$ (see [6, p. 573]). It is well known [6, Chapter 7] that $A$ commutes with $T$, $\sigma\left(T_{A X}\right)=\tau$, and $\sigma\left(T_{(I-A) X}\right)=\sigma(T) \backslash \tau$, and therefore $q\left(T_{A X}\right) \geq t>t_{1}$ and $r\left(T_{(I-A) X}\right) \leq t<t_{2}$. This shows that $T$ is radially super-decomposable, and the assertion follows from Theorem 4.1.

The following is an immediate consequence of Theorem 4.2.

Corollary 4.3. If $T$ is an operator in $\mathscr{L}(X)$ such that $\sigma(T)$ is included in a countable union of circles with centers at the origin, then $T$ is power-regular.

Corollary 4.3 implies that all operators considered in [2] which are annihilated by a nonzero analytic function are power-regular, since by $[2$, Theorem $3(\mathrm{a})$ ], if $T$ is such an operator, then the set $\{|\lambda| ; \lambda \in \sigma(T)\}$ is countable. This includes, in particular, operators of class $C_{0}$, that is, completely nonunitary contractions in Hilbert space, which are annihilated by a nonzero bounded analytic function in the unit disc. For this class power-regularity follows also from Theorem 3.1, since by a result of Foias [8], operators of class $C_{0}$ are decomposable.

\section{FINITE-DIMENSIONAL SPACES}

If $X$ is finite dimensional, then every operator in $\mathscr{L}(X)$ has finite spectrum and hence is power-regular by Corollaries 3.2 or 4.3 . This also follows from $[11$, p. 116] where the more general case of compact operators is considered. In that proof the Jordan canonical form is used. We give here two direct and elementary proofs.

Theorem 5.1. If $X$ is finite dimensional and $T$ is in $\mathscr{L}(X)$, then for all $x$ in $X$,

$$
\lim _{n \rightarrow \infty}\left\|T^{n} x\right\|^{1 / n}=r_{x}(T) \text {. }
$$

First proof. Let $x \in X$. By considering the restriction of $T$ to the subspace spanned by the vectors $x, T x, T^{2} x, \ldots$, we may assume that $x$ is a cyclic vector for $T$. Let $\lambda$ be an eigenvalue of $T$ such that $|\lambda|=r(T)$, and let $v$ be a corresponding unit eigenvector. Since $x$ is a cyclic vector for $T$, there exists a polynomial $p$ such that $p(T) x=v$, and therefore for every positive integer $n$,

$$
(r(T))^{n}=|\lambda|^{n}=\left\|p(T) T^{n} x\right\| \leq\|p(T)\|\left\|T^{n} x\right\| .
$$

Hence noticing that $p(T) \neq 0$ (since $v \neq 0)$, we obtain that

$$
r(T) \leq \liminf _{n \rightarrow \infty}\left(\|p(T)\|^{1 / n}\left\|T^{n} x\right\|^{1 / n}\right)=\liminf _{n \rightarrow \infty}\left\|T^{n} x\right\|^{1 / n} .
$$

Combining this with the fact that

$$
\limsup _{n \rightarrow \infty}\left\|T^{n} x\right\|^{1 / n} \leq \lim _{n \rightarrow \infty}\left\|T^{n}\right\|^{1 / n}=r(T),
$$

the assertion follows.

Second proof. Assume again that $x$ is a cyclic vector for $T$. Let $\mathscr{A}$ be the algebra generated in $\mathscr{L}(X)$ by $T$ and the identity operator, that is, $\mathscr{A}=$ $\operatorname{span}\left\{T^{n} ; n=0,1, \ldots\right\}$. Consider the linear mapping $L: \mathscr{A} \rightarrow X$ defined by 
$L(S)=S x, S \in \mathscr{A}$. Since $x$ is a cyclic vector for $T, L$ is an isomorphism between the finite-dimensional Banach spaces $\mathscr{A}$ and $X$. Therefore, there exists a constant $c>0$ such that

$$
c^{-1}\|S\| \leq\|S x\| \leq c\|S\|
$$

for all $S$ in $\mathscr{A}$; hence in particular,

$$
c^{-1}\left\|T^{n}\right\| \leq\left\|T^{n} x\right\| \leq c\left\|T^{n}\right\|, \quad n=1,2, \ldots .
$$

This implies that

$$
\lim _{n \rightarrow \infty}\left\|T^{n} x\right\|^{1 / n}=\lim _{n \rightarrow \infty}\left\|T^{n}\right\|^{1 / n}=r(T),
$$

and the proof is complete.

Remarks. (1) The second proof is valid also for finite-dimensional spaces over the real field (in this case the spectral radius of an operator is defined by Gelfand's formula).

(2) For general $X$ and all $T$ in $\mathscr{L}(X)$, it is easily verified that for every $t>0$, the set $\{x \in X ; r(x, T)<t\}$ is a linear space (which is not generally closed in $X)$. Hence if $X$ is finite dimensional and $T$ is in $\mathscr{L}(X)$, then $\lim _{n \rightarrow \infty}\left\|T^{n} x\right\|^{1 / n}=r(T)$ for all $x$ in $X$, except (if $r(T)>0$ ) for $x$ in the subspace $\{x \in X ; r(x, T)<r(T)\}$. Thus, in particular, this equality holds with probability one (with respect to normalized area measure) for $x$ in the Euclidean unit sphere of $X$.

\section{AdDitional FACTS AND PROBLEMS}

It is easily verified that power-regularity is preserved by similarity and (finite) direct sums but is not in general preserved by sums and products. In fact, every Hilbert space operator $A$ is the sum of two power-regular operators $\frac{1}{2}\left(A+A^{*}\right)$ and $\frac{1}{2}\left(A-A^{*}\right)$ (recall that selfadjoint operators are power-regular), and every weighted shift is the product of a diagonal operator and an isometry (which are clearly power-regular), but as already mentioned in $\S 1$, there exist weighted shifts which are not power-regular. For commuting operators the situation is less clear.

Problem 1. Are the sum and product of two commuting power-regular operators also power-regular?

We show next that power-regularity is not preserved in general by adjoint operators. Consider the unilateral shift $S$ on $l^{2}$ (that is, $S e_{n}=e_{n+1}, n=$ $0,1, \ldots$, where $\left\{e_{n}\right\}_{n=0}^{\infty}$ is the standard orthonormal basis in $l^{2}$ ). Since $S$ is an isometry, it is power-regular. We claim that its adjoint $S^{*}$ (the backward shift) is not power-regular. To see this, consider the sequence $\left\{a_{n}\right\}_{n=0}^{\infty}$ in $l^{2}$ defined by $a_{0}=1$ and $a_{n}=\exp \left(-2^{k}\right), 2^{k-1} \leq n<2^{k}, k=1,2, \ldots$. Then $x=\left\{\left(a_{n}^{2}-a_{n-1}^{2}\right)^{1 / 2}\right\}_{n=0}^{\infty}$ is in $l^{2}$, and $\left\|S^{* n} x\right\|=a_{n}, n=1,2, \ldots$. Since the sequence $\left\{a_{n}^{1 / n}\right\}_{n=1}^{\infty}$ is not convergent, this shows that $S^{*}$ is not power-regular.

It is clear that power-regularity is preserved by restrictions to invariant subspaces. Hence, for example, power-regularity of subnormal operators follows from that of normal operators. On the other hand, the preceding example implies that power-regularity is not preserved in general by passing to quotient spaces. In fact, if $T$ is the unitary operator on $l^{2}(\mathbb{Z})$ defined by $T\left\{a_{n}\right\}_{n=-\infty}^{\infty}=$ 
$\left\{a_{n+1}\right\}_{n=-\infty}^{\infty}$, then the subspace $M$, which consists of all sequences $\left\{c_{n}\right\}_{n=-\infty}^{\infty}$ in $l^{2}(\mathbb{Z})$ such that $c_{n}=0$ for $n \geq 0$, is in $\operatorname{Lat}(T)$, and $T^{M}$ can be identified in an obvious way with the backward shift $S^{*}$.

For all operators $T$ in $\mathscr{L}(X)$ for which power-regularity was proved in the preceding sections, the equality

$$
\lim _{n \rightarrow \infty}\left\|T^{n} x\right\|^{1 / n}=r_{x}(T)
$$

was also established for all $x$ in $X$. We do not know whether this is always the case.

Problem 2. Assume that $T$ is a power-regular operator in $\mathscr{L}(X)$. Is the above equality satisfied for all $x$ in $X$ ?

For general operators the equality is not always true, even if the limit on the left-hand side exists. To see this, consider again the backward shift $S^{*}$ on $l^{2}$, and let $x$ denote the sequence $\{1 /(n+1) !\}_{n=0}^{\infty}$ in $l^{2}$. It follows from [10, p. 282] that $x$ is a cyclic vector for $S^{*}$, and therefore $r_{x}\left(S^{*}\right)=r\left(S^{*}\right)=1$. On the other hand, a simple estimate shows that

$$
\left\|S^{* n} x\right\| \leq \frac{1}{n !}, \quad n=1,2, \ldots,
$$

and therefore $\lim _{n-\infty}\left\|S^{* n} x\right\|^{1 / n}=0$. Hence the equality is not satisfied in this case.

It follows from Corollary 4.3 that every operator whose spectrum is included in a circle with center at the origin is power regular. We do not know whether the same is true for operators with spectrum included in a smooth curve, even for curves of very special type.

Problem 3. Is every operator in $\mathscr{L}(X)$ with spectrum included in a circle or in the real line power-regular?

We conclude with some comments about power-regular Banach algebras. It is clear that a closed subalgebra of a power-regular Banach algebra is also powerregular. Thus, by Corollary 3.3, every closed subalgebra of a commutative semisimple regular Banach algebra is power-regular. Also, for a general commutative Banach algebra $B$ with identity, one can show that for every $y$ in $B$ whose Gelfand transform does not vanish on any nonempty open subset of the maximal ideal space of $B$, and for all $x$ in $B$,

$$
\lim _{n \rightarrow \infty}\left\|x^{n} y\right\|^{1 / n}=r(x)
$$

(where $r(x)$ denotes the spectral radius of $x$ ). Thus, in particular, if every nonzero element $y$ of $B$ has this property, then $B$ is power-regular. Therefore, every Banach algebra with identity of analytic functions on some domain in the complex plane, which is dense in its maximal ideal space, is power-regular.

In a lecture at the Banach center we raised the question whether every commutative semisimple Banach algebra with identity is power-regular. Vladimir Müller constructed an example which shows that this is not the case.

\section{ACKNOWLEDGMENT}

I wish to express my thanks to Vladimir Müller and Andrej Soltysiak for stimulating conversations. 


\section{REFERENCES}

1. E. Albrecht, On decomposable operators, Integral Equations and Operator Theory 2 (1979), $1-10$.

2. A. Atzmon, Operators which are annihilated by analytic functions and invariant subspaces, Acta Math. 144 (1980), 27-63.

3. B. Beauzamy, Introduction to operator theory and invariant subspaces, North-Holland, Amsterdam, 1988.

4. I. Colojoara and C. Foiaş, Theory of generalized spectral operators, Gordon and Breach, New York, 1968.

5. J. Daneš, On local spectral radius, Časopis Pešt. Mat. 112 (1987), 177-187.

6. N. Dunford and J. T. Schwartz, Linear operators, Part I, Interscience, New York, 1958.

7. I. Erdelyi and R. Lange, Spectral decompositions on Banach spaces, Lecture Notes in Math., vol. 623, Springer, Berlin, 1977.

8. C. Foias, The class $C_{0}$ in the theory of decomposable operators, Rev. Roumaine Math. Pures Appl. 14 (1969), 1433-1440.

9. J. D. Gray, Local analytic extensions of the resolvent, Pacific J. Math. 27 (1968), 305-324.

10. P. R. Halmos, A Hilbert space problem book, Van Nostrand, New York, 1967.

11. M. A. Krasnosel'skii et al., Approximate solutions of operator equations, Wolters-Noordhoff, Gröningen, 1972.

12. K. B. Laursen and M. M. Neumann, Decomposable operators and automatic continuity, J. Operator Theory 15 (1986), 33-51.

13. V. Müller, Local spectral radius formula for operators in Banach spaces, Czechoslovak Math. J. 38 (1988), 726-729.

School of Mathematical Sciences, Sackler faculty of Exact Sciences, Tel Aviv UniVERSiTy, Tel AVIV 69978, IsRAEL

E-mail address: aatzmon@math.tau.ac.il 